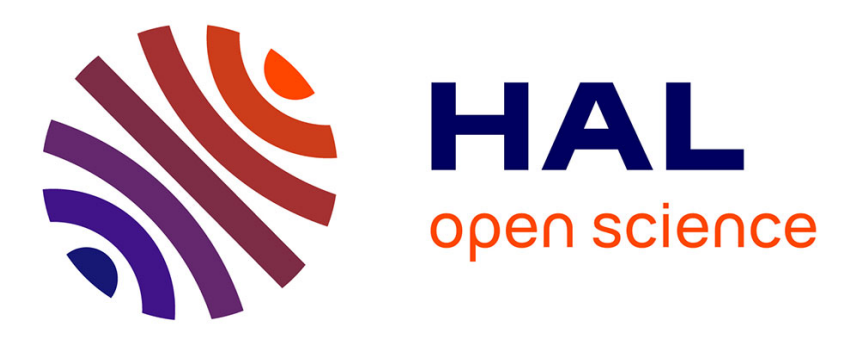

\title{
Photoacoustic guidance of high intensity focused ultrasound with selective optical contrasts and time-reversal
}

Arik Funke, Jean-François Aubry, Mathias Fink, Albert-Claude Boccara, Emmanuel Bossy

\section{To cite this version:}

Arik Funke, Jean-François Aubry, Mathias Fink, Albert-Claude Boccara, Emmanuel Bossy. Photoacoustic guidance of high intensity focused ultrasound with selective optical contrasts and time-reversal. Applied Physics Letters, 2009, 94 (5), pp.054102. 10.1063/1.3077018 . hal-02481867

\section{HAL Id: hal-02481867 https://hal.science/hal-02481867}

Submitted on 17 Feb 2020

HAL is a multi-disciplinary open access archive for the deposit and dissemination of scientific research documents, whether they are published or not. The documents may come from teaching and research institutions in France or abroad, or from public or private research centers.
L'archive ouverte pluridisciplinaire HAL, est destinée au dépôt et à la diffusion de documents scientifiques de niveau recherche, publiés ou non, émanant des établissements d'enseignement et de recherche français ou étrangers, des laboratoires publics ou privés. 


\section{Photoacoustic guidance of high intensity focused ultrasound with selective optical contrasts and time-reversal}

Cite as: Appl. Phys. Lett. 94, 054102 (2009); https://doi.org/10.1063/1.3077018

Submitted: 14 December 2008 . Accepted: 08 January 2009. Published Online: 03 February 2009

Arik R. Funke, Jean-François Aubry, Mathias Fink, Albert-Claude Boccara, and Emmanuel Bossy

\section{ARTICLES YOU MAY BE INTERESTED IN}

Photoacoustic imaging in biomedicine

Review of Scientific Instruments 77, 041101 (2006); https://doi.org/10.1063/1.2195024

Time reversal of photoacoustic waves

Applied Physics Letters 89, 184108 (2006); https://doi.org/10.1063/1.2382732

Iterative reconstruction algorithm for optoacoustic imaging

The Journal of the Acoustical Society of America 112, 1536 (2002); https://

doi.org/10.1121/1.1501898

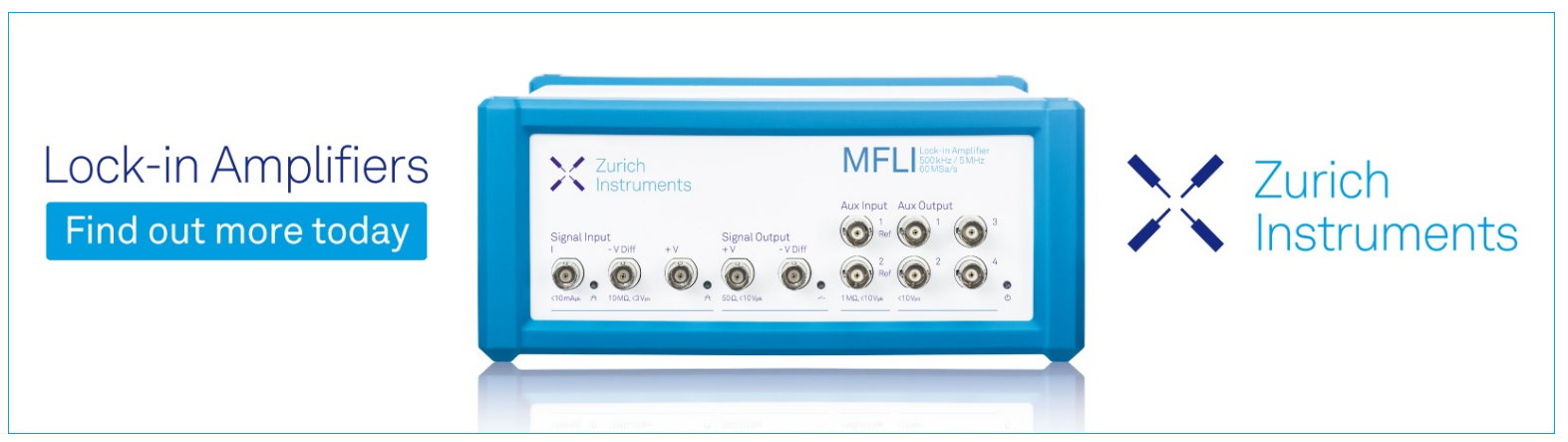




\title{
Photoacoustic guidance of high intensity focused ultrasound with selective optical contrasts and time-reversal
}

\author{
Arik R. Funke, Jean-François Aubry, Mathias Fink, Albert-Claude Boccara, and \\ Emmanuel Bossy ${ }^{\text {a) }}$ \\ Institut Langevin, ESPCI ParisTech, CNRS UMR 7587, 10 rue Vauquelin, 75231 Paris Cedex 05, France
}

(Received 14 December 2008; accepted 8 January 2009; published online 3 February 2009)

\begin{abstract}
The authors present a method of focusing high intensity ultrasound by time-reversing the photoacoustic response of an optically selective target in a nonselective background. The target's photoacoustic response was isolated from the background by subtracting the photoacoustic waveforms obtained at different optical wavelengths and convolved with a continuous signal. It was found that the focus produced was comparable in quality to that obtained by delay-law beam-forming. The method holds the promise of allowing precise targeting of high intensity focused ultrasound on nonechogenic targets, in moving environments, independently of the presence of aberrating layers. (C) 2009 American Institute of Physics. [DOI: 10.1063/1.3077018]
\end{abstract}

High intensity focused ultrasound (HIFU) allows the noninvasive treatment of tumors by raising the tissue temperature locally to induce apoptosis and thermal necrosis without damage to surrounding tissues. ${ }^{1,2}$ However, the ability to focus ultrasound is essential to ensure sufficiently localized energy deposition and thus limit the thermal necrosis to the target region. This requirement becomes a limiting factor to the application of the method where the target region lies behind an ultrasonically aberrating layer such as an irregular layer of fat tissue, intracostally, or intracranially. Other challenges arise from the motion of the target, e.g., due to respiration, or from nonechogenic targets leading to uncertainty in the position of the target.

One approach that has been shown to allow compensating very effectively for aberration effects is time reversal, or in the continuous wave case, phase-conjugation methods. Given that the wave equation exhibits time-reversal invariance, if a point source at the desired focus can be generated and its signal be acquired after traversing the aberrating layer and reemitted temporally reversed, the reemitted signal will reconverge at the position of the point source.,

One essential requirement for time-reversal methods is the existence of a point source at the desired focus. Depending on the application, various methods have been developed to generate such a point source. An early, and to date still the most effective, approach, was to implant a needle hydrophone intracranially to serve as a point source via reciprocity. ${ }^{5,6}$ While this approach can be minimally invasive, a noninvasive technique would be preferable especially in the case of the brain. Alternatives are the generation of a cavitation bubble ${ }^{7}$ or the simulation of a point source in a virtual model of the acoustic medium that was acquired by $\mathrm{x}$-ray computed tomography. ${ }^{8-10}$ The first alternative may suffer from the uncertainty in the axial position of the generated bubble. The second one is limited by the duration of the required computations and the need for the alignment between the virtual model and real world with millimetric precision. Various methods have been developed for the challenge of motion compensation, e.g., based on cross cor-

\footnotetext{
a) Author to whom correspondence should be addressed. Electronic mail: emmanuel.bossy@espci.fr.
}

relation of the acoustic speckle pattern. ${ }^{10,11}$ For targeting of nonechogenic targets, multimode systems with a shared coordinate system are currently necessary for precise treatment, e.g., HIFU under magnetic resonance imaging guidance. ${ }^{12}$

In this letter, a method of HIFU guidance is proposed. It potentially offers convenient solutions to the problems of aberration correction, motion compensation, and nonechogenic targets via photoacoustic generation in the target.

Photoacoustic generation refers to the generation of acoustic waves by an optical absorber that undergoes thermoelastic expansion upon absorption of a light pulse. The resulting photoacoustic pressure field $p$ is given by

$$
\Delta p-\frac{1}{c_{s}^{2}} \frac{\partial^{2} p}{\partial t^{2}}=-\frac{\beta}{c_{p}} \frac{\partial H}{\partial t},
$$

where $H(\vec{r}, t)=\mu_{a}(\vec{r}) \phi(\vec{r}, t)$ and where $\Delta$ is the Laplacian, $H$ is the density of absorbed optical energy per unit time, $c_{s}$ is the adiabatic sound speed, $\beta$ is the isobaric thermal expansion coefficient, $c_{p}$ is the specific heat, $\mu_{a}$ is the optical absorption coefficient, and $\phi$ is the fluence rate. In an earlier work, we showed that the photoacoustic signal from a black sphere suspended in an optically scattering medium can be used with time-reversal methods to correct for aberrating layers. ${ }^{13}$ However, only a single optical absorber was present. This is in stark contrast to a biological environment where numerous different chromophores and structures, such as, for example, blood vessels, may produce strong photoacoustic signals compared to the target of interest.

Photoacoustic spectroscopy has been used with endogenous contrasts to access functional tissue parameters. ${ }^{14,15}$ In this work it is proposed to use photoacoustic signals for the guidance of HIFU treatment by attaching an exogenous optically selective contrast agent to the target region and using the difference in the photoacoustic waveforms generated at different optical wavelengths to extract targeting information. The proposed method holds the promise of allowing to learn to focus on a target region beyond aberrating layers and of allowing to compensate for target motion, thanks to the rapidity of the method; time-reversal correction is physically limited by the total time of flight (typically $67 \mu \mathrm{s}$ for a target located $5 \mathrm{~cm}$ in the tissue). Given that the focusing 


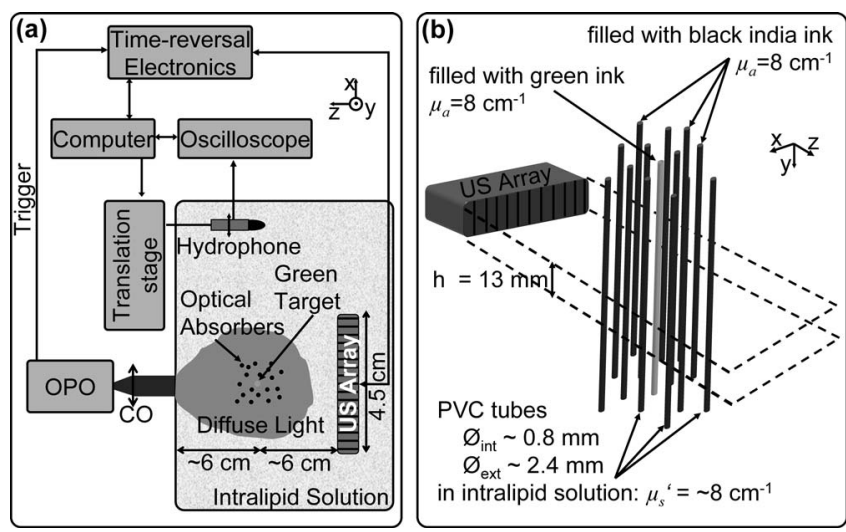

FIG. 1. Experimental setup. CO denotes collimation optics.

process is based fundamentally on optical absorption, the targets need not be echogenic. In the following a proof-ofconcept is presented with tubes filled with ink used as optical absorbing structures, embedded in a phantom that mimics specifically the optical properties of biological tissue.

The experimental setup is shown in Fig. 1. The tubes were made of optically transparent PVC, $50 \mathrm{~mm}$ in height with an internal diameter and wall thickness of $0.8 \mathrm{~mm}$ each. They were arranged vertically and quasirandomly in a $50 \mathrm{~mm}$ (depth) $\times 80 \mathrm{~mm}$ (transverse) area with a spacing of typically $17 \mathrm{~mm}$. All but one of these tubes were filled with a solution of black India ink with $\mu_{a}=8.0 \mathrm{~cm}^{-1}$ at $\lambda_{1}$ $=672 \mathrm{~nm}$ and $\mu_{a}=7.3 \mathrm{~cm}^{-1}$ at $\lambda_{2}=730 \mathrm{~nm}$. One of the tubes, located roughly at the center, was filled with a solution of green India ink with $\mu_{a}=8.0 \mathrm{~cm}^{-1}$ at $\lambda_{1}$ and $\mu_{a}$ $=0.2 \mathrm{~cm}^{-1}$ at $\lambda_{2}$. The tubes filled with black ink simulate nonselective optical absorption, e.g., from blood vessels. The tube filled with green ink serves as optically selective target.

The set of tubes was placed in an optically transparent aquarium filled with 5.621 of de-ionized and degassed water in which $450 \mathrm{ml}$ of $10 \%$ intralipid was diluted. The resulting solution has a reduced scattering coefficient comparable to that of biological tissue, $\mu_{s}^{\prime}=8.9 \mathrm{~cm}^{-1}$ at $\lambda_{1}$ and $\mu_{s}^{\prime}$ $=8.1 \mathrm{~cm}^{-1}$ at $\lambda_{2}$. ${ }^{16}$ The tubes are arranged such that the tube filled with green ink was roughly $60 \mathrm{~mm}$ from the aquarium wall and $60 \mathrm{~mm}$ from the transducer surface. This tissuemimicking phantom was then illuminated through the aquarium wall with $5 \mathrm{~ns}$ light pulses at $\lambda_{1}$ and $\lambda_{2}$ with an energy of $100 \mathrm{~mJ}$ in a beam with a diameter of approximately $5 \mathrm{~mm}$ with a repetition rate of $10 \mathrm{~Hz}$. To generate the light a Surelite OPO Plus driven by a Surelite II-10 (Continuum, CA) was used.

The generated photoacoustic waveforms were acquired with a commercial 64-element linear imaging probe from Vermon, France with $1.5 \mathrm{MHz}$ nominal central frequency, a $13 \times 45 \mathrm{~mm}^{2}$ aperture, and $60 \mathrm{~mm}$ prefocusing in elevation. The probe was connected to a time-reversal system (Open System, Lecoeur Electronique, France) with 64 independently programmable channels with a sampling frequency of $80 \mathrm{MHz}$ triggered by the laser. The data were transferred and then processed on a computer.

At this stage, neither the ultrasonic probe nor the timereversal electronics used in this experiment could sustain the energies necessary for HIFU. Thus to test the ability to guide "HIFU mode" signals, $45 \mu$ s sinusoidal signals were emitted with the maximum available energy for our system on all channels. To test the ability to guide HIFU mode signals, the tubes were removed from the aquarium and a hydrophone (HGL-400, Onda, CA) was introduced. With the aid of ultrasonic imaging the hydrophone was positioned at the location of the target tube relative to the ultrasonic probe and scanned transversally to the ultrasonic probe, while the ultrasonic probe emitted the time-reversed signals.

Due to the limits on the permissible energy density of the illumination of biological tissue and the strong scattering the light experiences, the energy available for absorption by optical contrasts embedded at several centimeters in depth is scarce. To increase the SNR of the data, the acquired photoacoustic signals were averaged over 86 acquisitions, and the result was filtered with the bandwidth of the transducer.

Moreover, the fluence rate decreases with increasing distance from the point of light injection. In consequence, as the amplitude of photoacoustic signals is proportional to the absorbed energy for short pulses, identical optical absorbers at different depths produce photoacoustic signals of different amplitudes. To compensate for the unknown attenuation of the fluence rate, a compensation function was calculated using regression on the amplitude of the photoacoustic waveforms as a function of depth; assuming that light injection can be considered as a point source in the scattering medium, the fluence rate decays as $r^{-1} \exp \left(-\mu_{\mathrm{eff}} r\right)$, where $r$ is the distance from the point of light injection and $\mu_{\mathrm{eff}}$ an effective light attenuation coefficient.

For our data analysis we used the maximum value of each acquisition line, thereby treating the distance coordinate $r$ as equivalent to the depth from the aquarium wall. The effect of the attenuation on the photoacoustic waveforms can then be compensated for by normalizing with the result of the regression. Figure 2 shows beam-formed photoacoustic images obtained from the photoacoustic waveforms at different stages of the signals processing. Figure $2(a)$ is based on the photoacoustic waveforms obtained at $\lambda_{1}$ (with background and target) after averaging and filtering, while Fig. 2(b) is based on the photoacoustic waveforms obtained at $\lambda_{1}$ after additionally applying the compensation function. In this experiment $\mu_{\mathrm{eff}}=0.7 \mathrm{~cm}^{-1}$ was found for both $\lambda_{1}$ and $\lambda_{2}$.

The attenuation-compensated photoacoustic waveforms obtained at the two different optical wavelengths were then subtracted. Characteristics that were identical at both wavelengths, i.e., photoacoustic signals from optically nonselective absorbers, cancelled and only the differences remained, i.e., the signals from the optically selective target. This result shall be referred to in the following as "difference waveforms." Comparing Fig. 2(b) based on waveforms obtained at $\lambda_{1}$ (with background and target) and Fig. 2(c) based on waveforms obtained $\lambda_{2}$ (with background but without target), it is clear that in Fig. 2(b) the target is missing at the center of the image. Figure 2(d) shows the result based on the difference waveforms. The residual signal remaining at the positions of the nonselective absorbers can be explained by the fact that the PVC tubes used to contain the ink solutions are not entirely transparent but absorb differently at $\lambda_{1}$ and $\lambda_{2}$.

To construct the signal for the verification of the ability to focus HIFU mode ultrasound, a sinusoidal signal of $45 \mu \mathrm{s}$ duration was created for all emission channels at the frequency that corresponds to the maximum of the difference waveforms, $f=1.16 \mathrm{MHz}$. The emission signal was defined by convolving either with the time-reversed photoacoustic waveforms obtained at $\lambda_{1}$ or with the time-reversed differ- 

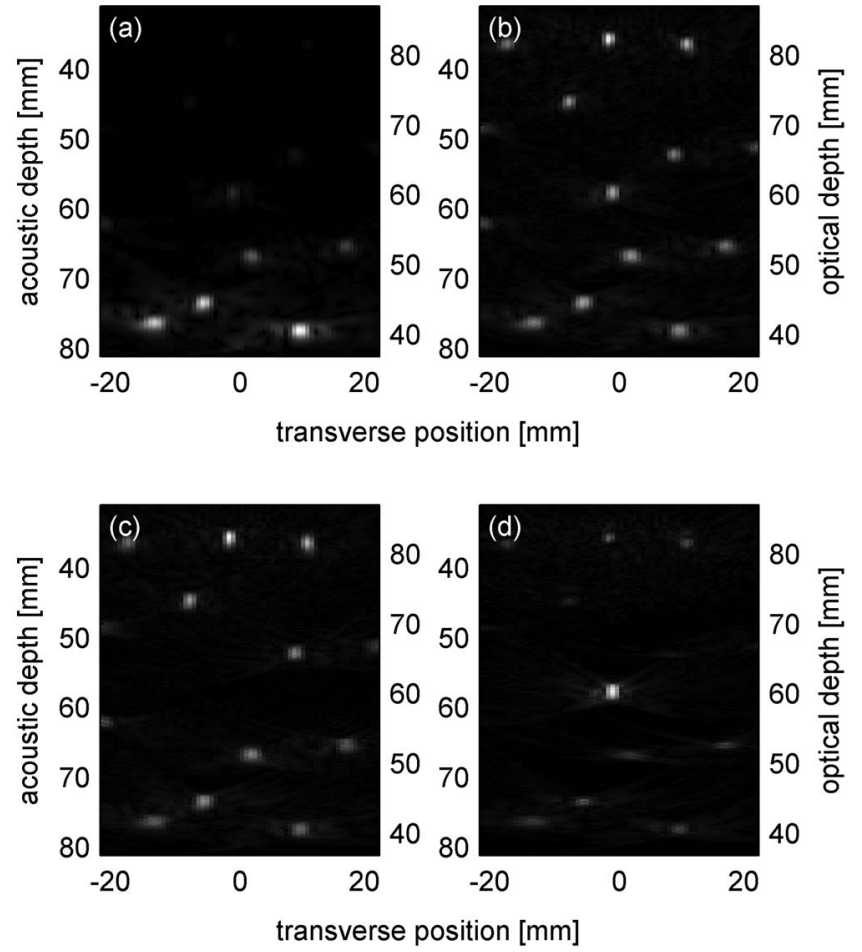

FIG. 2. Beam-formed images based on the waveforms at different steps in the signal processing. (a) is based on the waveforms obtained at $\lambda_{1}$ after averaging and filtering but without depth compensation, the data of (b) were additionally compensated for depth, (c) is the analog to (b) but for $\lambda_{2}$, and (d) is based on the difference waveforms: the isolated target is clearly visible. All images are on a linear gray scale and have been normalized to their respective maximum value.

ence waveforms. Focusing was verified by scanning a hydrophone in the plane of the target normal to the acoustic axis.

The effectiveness of using an optically selective absorber embedded in an environment with multiple nonselective absorbers for the targeting of ultrasound is illustrated in Fig. 3.
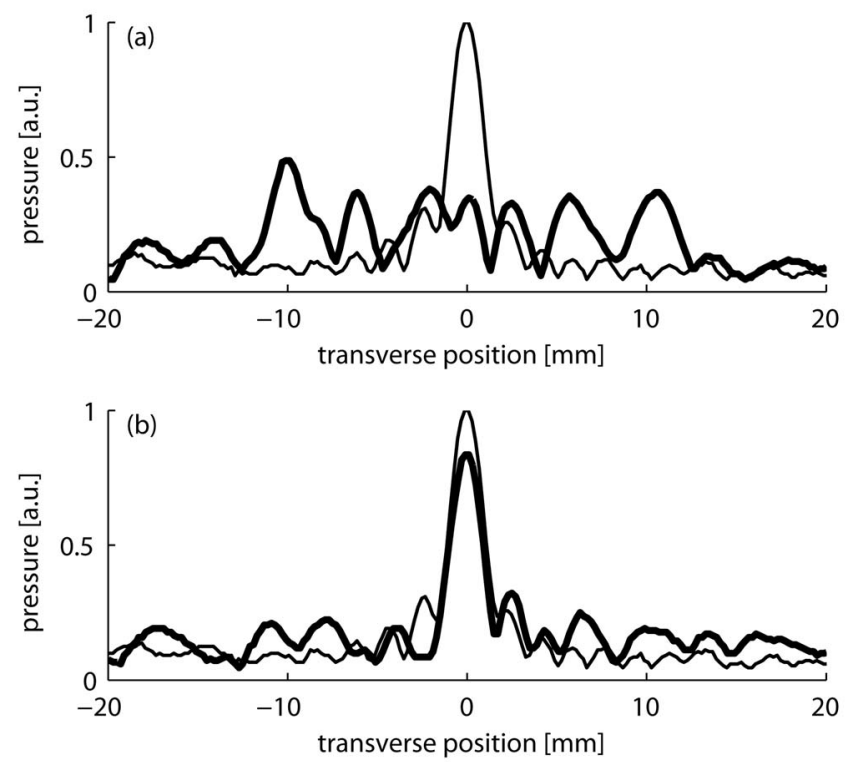

FIG. 3. Hydrophone scans of the focus produced by a beam-formed sinusoidal signal (fine line) and (a) by reemitting a sinusoidal signal focused by use of the phase of the photoacoustic signals obtained at $672 \mathrm{~nm}$ (bold line), i.e., with target and background, and (b) by reemitting a sinusoidal signal focused by use of the phase of the photoacoustic signal from the isolated target (bold line).
The figure plots the acoustic pressure amplitude measured with a hydrophone in the plane of the target. The position of the optically selective target corresponds to $0 \mathrm{~mm}$. To serve as a basis for comparison, the fine line corresponds to the focus obtained by beam forming the emitted signal with a conventional delay law for homogeneous media. In Fig. 3(a) the bold line illustrates the pressure amplitude distribution obtained by convolving the sinusoidal signal with the timereversed photoacoustic waveforms obtained at $\lambda_{1}$. They contain the waveforms from the selective and nonselective absorbers and consequently produce foci on the selective and the various nonselective absorbers, spreading the acoustic energy over the entire phantom. In Fig. 3(b) the bold line plots the focus produced by convolving the sinusoidal signal with the time-reversed difference waveforms. It contains essentially the isolated photoacoustic waveforms of the optically selective target. The figure clearly shows that the full width half maximum of the focus obtained from conventional delay-law beam-forming and from convolving with the photoacoustic waveform is quasi identical.

In conclusion, a method for guiding HIFU mode ultrasound with a selective-optical contrast agent and timereversal was proposed and its feasibility demonstrated with a tissue phantom and a commercial imaging probe. This method holds the promise of allowing overcoming three significant challenges in HIFU treatment: aberration correction, movement compensation, and targeting of nonechogenic targets. An important remaining challenge is the detection the photoacoustic signals with sufficient SNR with a transducer that can withstand the sustained powers necessary for HIFU treatment.

The authors gratefully acknowledge the support of the Centre National de la Recherche Scientifique and the Agence Nationale de la Recherche (Grant No. JC07-195015).

${ }^{1}$ F. Wu, Z. B. Wang, W. Z. Chen, J. Z. Zou, J. Bai, H. Zhu, K. Q. Li, F. L. Xie, C. B. Jin, H. B. Su, and G. W. Gao, Ultrasound Med. Biol. 30, 245 (2004).

${ }^{2}$ R. O. Illing, J. E. Kennedy, F. Wu, G. R. ter Haar, A. S. Protheroe, P. J. Friend, F. V. Gleeson, D. W. Cranston, R. R. Phillips, and M. R. Middleton, Br. J. Cancer 93, 890 (2005).

${ }^{3}$ M. Fink, IEEE Trans. Ultrason. Ferroelectr. Freq. Control 39, 555 (1992).

${ }^{4}$ M. Fink and C. Prada, Inverse Probl. 17, R1 (2001).

${ }^{5}$ M. Tanter, J. L. Thomas, and M. Fink, J. Acoust. Soc. Am. 103, 2403 (1998).

${ }^{6}$ M. Pernot, J. F. Aubry, M. Tanter, A. L. Boch, F. Marquet, M. Kujas, D. Seilhean, and M. Fink, J. Neurosurg. 106, 1061 (2007).

${ }^{7}$ M. Pernot, G. Montaldo, M. Tanter, and M. Fink, Appl. Phys. Lett. 88, 034102 (2006).

${ }^{8}$ J. F. Aubry, M. Tanter, M. Pernot, J. L. Thomas, and M. Fink, J. Acoust. Soc. Am. 113, 84 (2003).

${ }^{9}$ G. T. Clement and K. Hynynen, Phys. Med. Biol. 47, 1219 (2002).

${ }^{10}$ M. Tanter, M. Pernot, J. F. Aubry, G. Montaldo, F. Marquet, and M. Fink, Int. J. Hyperthermia 23, 413 (2007).

${ }^{11}$ M. Pernot, M. Tanter, and M. Fink, Ultrasound Med. Biol. 30, 1239 (2004).

${ }^{12}$ C. M. C. Tempany, E. A. Stewart, N. McDannold, B. J. Quade, F. A. Jolesz, and K. Hynynen, Radiology 226, 897 (2003).

${ }^{13}$ E. Bossy, K. Daoudi, A. C. Boccara, M. Tanter, J. F. Aubry, G. Montaldo, and M. Fink, Appl. Phys. Lett. 89, 184108 (2006).

${ }^{14}$ E. V. Savateeva, A. A. Karabutov, S. V. Solomatin, and A. A. Oraevsky, Proc. SPIE 4618, 63 (2002).

${ }^{15}$ X. D. Wang, X. Y. Xie, G. N. Ku, and L. H. V. Wang, J. Biomed. Opt. 11 024015 (2006).

${ }^{16}$ H. J. Vanstaveren, C. J. M. Moes, J. Vanmarle, S. A. Prahl, and M. J. C. Vangemert, Appl. Opt. 30, 4507 (1991). 